\title{
The platelet-derived growth factor (PDGF) family of tyrosine kinase receptors: a Kit to fix the beta cell?
}

\author{
M. Welsh
}

Received: 9 May 2012 / Accepted: 24 May 2012 / Published online: 14 June 2012

(C) Springer-Verlag 2012

\begin{abstract}
Overexpression of c-Kit has recently been shown to ameliorate beta cell function by increasing the beta cell mass and insulin secretion, thus counteracting the deleterious effects of a high-fat diet on glucose homeostasis. The c-Kit-dependent effects are due to enhanced Akt activity that phosphorylates and inhibits glycogen synthase kinase $3 \beta$ (GSK3 $\beta$ ), thereby increasing the expression of numerous genes that promote insulin production and cell proliferation. Regulating the c-Kit/Akt/GSK3 $\beta$ pathway may provide novel means for improving beta cell function in type 2 diabetes.
\end{abstract}

Keywords Beta cell · c-Kit · Diabetes · Glucose tolerance . Proliferation · Tyrosine kinase receptors

\section{Abbreviations \\ PDGF Platelet-derived growth factor \\ FLT3 Fms-like tyrosine kinase 3 \\ SCF Stem cell factor \\ GSK3 $\beta \quad$ Glycogen synthase kinase $3 \beta$}

The platelet-derived growth factor (PDGF) receptor family of receptor tyrosine kinases (class III) consists of the PDGF receptors and the receptors known as c-Kit, c-Fms and Fmslike tyrosine kinase 3 (FLT3). Whereas c-Fms and FLT3 primarily play a role in haematopoietic cells, c-Kit and the PDGF receptors have more diverse effects. c-Kit is of importance for the development of gametes, hematopoietic cells, melanocytes and mast cells [1], whereas the PDGF

\section{Welsh $(\triangle)$}

Department of Medical Cell Biology, Uppsala University,

Box 571, Husargatan 3,

75123, Uppsala, Sweden

e-mail: Michael.welsh@mcb.uu.se receptors are important for vascular mural cells, glial cells in the central nervous system and the formation of lung alveoli, intestinal villi and hair follicles [2]. These tyrosine kinase receptors have the ability to stimulate cell proliferation, increase survival and cause cellular differentiation and migration by signalling through the extracellular-signal regulated kinase (ERK) and phosphoinositide-3-kinase (PI3K) pathways. Several oncogenic mutants of these receptors and/or their corresponding ligands have been characterised.

The group of Dr Rennian Wang, at the University of Western Ontario in Canada, has for several years systematically pursued a line of research investigating the role of c-Kit in beta cells. These studies have culminated in the exciting story of the c-Kit-dependent rescue of beta cell failure, which is published in this issue of Diabetologia [3].

The importance of PDGF for beta cells has been known for many years and early studies indicated that PDGF stimulates beta cell replication [4], that this effect is promoted by overexpression of PDGF receptors [5] and that PDGF receptors are expressed in pancreatic epithelial cells during embryonic development [6]. Recently, an age-dependent decrease in PDGF receptor expression was described that diminished the proliferative potential of beta cells, and beta cell-specific inactivation of the gene encoding PDGF receptor $\alpha(P d g f r a)$ aggravated susceptibility to the beta cell toxin streptozotocin [7]. These results implicate PDGF receptors as potential targets for additional treatment regimens for diabetes. This could involve the administration of PDGF ligands and/or increased expression of PDGF receptors in beta cells.

Our understanding of the involvement of c-Kit in beta cell function has a more recent history. Expression of c-Kit was detected in embryonic pancreatic epithelial cells or adult islets, and the addition of the c-Kit ligand, stem cell factor SCF, to a culture of fetal islet-like structures increased 
their insulin content [6, 8-11]. Expression of c-Kit has been detected on both endocrine and non-endocrine epithelial cells. In addition, SCF or a c-Kit-activating antibody increased the proportion insulin-positive cells in human fetal clusters of epithelial cells that resemble islets $[12,13]$. Besides playing a role in beta cell development, c-Kit is also important for adult beta cell function. Male mice harbouring a c-Kit mutation have elevated blood glucose levels and impaired glucose tolerance [14]. They exhibit a reduced beta cell mass and insulin gene expression. Female mutant mice showed a less dramatic phenotype. Surprisingly, the addition of the glycogen synthase kinase $3 \beta$ (GSK $3 \beta$ ) inhibitor 1-azakenpaullone reversed the deleterious effect of the c-Kit mutant on glucose homeostasis, suggesting that the GSK3 $\beta$ pathway is responsible for the adverse effects [15].

The present investigation shows that elevated expression of c-Kit improves beta cell function, particularly in male mice [3]. The authors adopted a transgenic overexpression strategy allowing the rat insulin promoter to drive beta cell c-Kit expression. As expected, isolated islets exhibited c-Kit overexpression at the RNA and protein level. The mice had improved glucose tolerance, glucose-stimulated insulin secretion and islet insulin content, without showing differences in peripheral insulin sensitivity. The effects were partly due to an increased beta cell mass. Surprisingly, there was an increased mRNA content of various genes related to specific beta cell functions, such as $P d x 1$, Neurodl, Mafa, Pax6, Nkx2-2, Glut2 and Ins1 and Ins2. The levels of Gcg (encoding glucagon) and the Glp1r mRNA were also increased. Together, the findings point to changes in the beta cell phenotype that yield an improved insulin secretory capacity. The transgenic mice exhibited altered signalling characteristics, with elevated activity of Akt and increased levels of the cell cycle protein cyclin D1. The most important effects were observed when wild-type or $c$-Kit transgenic mice were placed on a high-fat diet. The transgenic mice were resistant to high-fat-diet-induced impaired glucose tolerance, gained less weight and had lower fasting blood glucose levels and increased glucose-stimulated insulin release. They also exhibited an increased beta cell mass and elevated gene expression of Pdx1, Mafa, Ins1/Ins2, Insr and Irs 1. Finally, the transgene partly reversed the deleterious phenotype of the c-Kit mutant with respect to glucose metabolism, clearly implicating c-Kit in the observed beneficial effects.

The increased beta cell mass is not surprising considering c-Kit stimulates cell replication in many systems. What is more puzzling is that the islets perform better, showing an increased secretory capacity and elevated expression of genes associated with the insulin secretory pathway. Is an increased beta cell mass a viable explanation for the secretory changes, allowing the cells to develop a more robust

Fig. 1 Schematic view of c-Kitdependent effects that promote beta cell replication and insulin secretion, thereby ameliorating beta cell function. Ligandstimulated c-Kit activates Akt (via PI3K), which inhibits GSK $3 \beta$ by an inhibitory phosphorylation. The GSK $3 \beta$ kinase regulates gene expression. Consequently, numerous changes in beta cell gene transcription follow its inhibition, including expression of the genes encoding the insulin receptor (Insr) and the IR docking protein IRS1 (Irs l). Increased expression and activity of these signalling components will cause further activation of Akt. In addition, increased expression of genes promoting glucose-stimulated insulin secretion, e.g. Pdxl, Mafa, Glut2 and Ins1/Ins2, and those increasing the beta cell mass (CcndI encoding cyclin D1) are observed under these conditions. IR, insulin receptor; PI3K, phosphoinositide 3-kinase

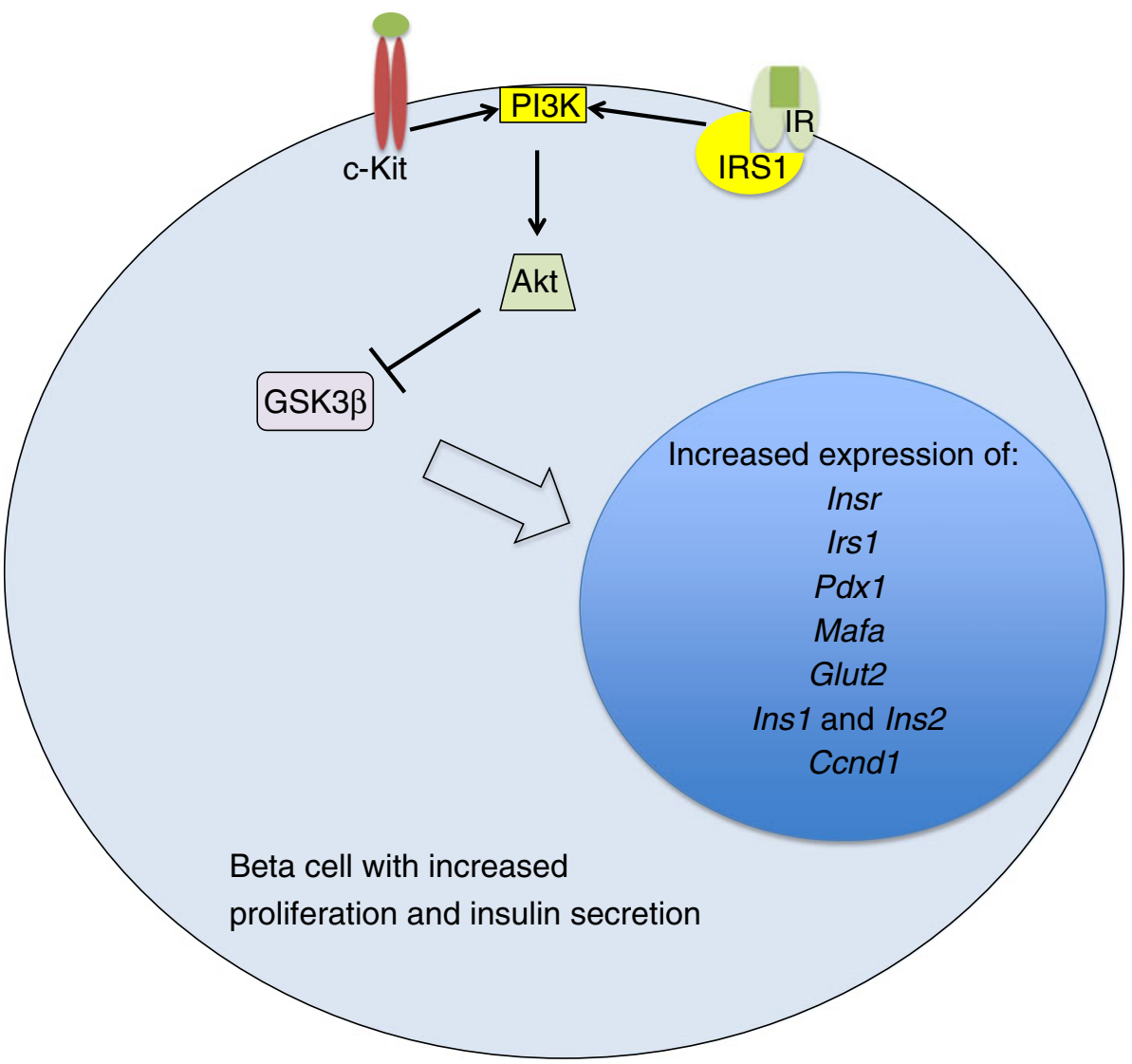


secretory phenotype over time, owing to less exposure to possible beta cell challenge or 'exhaustion'? There are other examples of transgenic models with an increased beta cell mass that exhibit an increased insulin secretory capacity [16, 17]. Conversely, there are examples of experimental models with an increased beta cell mass that do not show an increased insulin secretory capacity in response to glucose in vitro [18], leaving this issue unresolved. It seems plausible that certain changes occurring in the beta cell signal transduction signature promote both cell replication and secretory changes, whereas others stimulate only one of these functional aspects.

Increased Akt activity was noted [3], and this is an anticipated finding since SCF-stimulated c-Kit is known to activate Akt [1]. On the other hand, increased expression of the insulin receptor and IRS1 was also detected and, if these changes are translated into augmented signalling, they would also result in stimulated Akt (Fig. 1). One potential target downstream of Akt is forkhead box protein $\mathrm{O} 1$ (FOXO1), but this transcription factor was presently not investigated. Another target of Akt is GSK3 $\beta$ which becomes phosphorylated at an inhibitory site (Fig. 1). The importance of GSK $3 \beta$ in the present context is inferred from the finding that selective beta cell-specific ablation of the gene encoding GSK $3 \beta$ increases the beta cell mass, the insulin secretory response and resistance to the deleterious effects of a high-fat diet [19]. In addition, GSK3 $\beta$ was also found to regulate the expression of IRS1 and IRS2, providing another piece of the puzzle of which interactions are of relevance to the present study. As mentioned above, inhibition of the kinase by 1-azakenpaullone reversed the detrimental effects of the c-Kit mutation and GSK3 $\beta$ appears to be the common denominator for regulation of the beta cell mass and insulin secretion. Surprisingly, beta cell-specific Akt overexpression did not improve glucose stimulated insulin secretion [18], suggesting that other pathways besides Akt may be required for the complete phenotype. Interestingly, GSK3 $\beta$ activity potentially provides a mechanistic link to the type 2 diabetes susceptibility gene TCF7L2 [20], since GSK3 $\beta$ regulates the levels of $\beta$-catenin, a factor that forms a transcriptionally active complex with transcription factor 7-like 2 (TCF7L2) upon entry to the nucleus.

Using a rat insulin promoter to drive transgene expression will certainly overexpress the target gene in beta cells, but expression at other sites, including the hypothalamus, must be considered. There is currently no indication of possible off-target expression of the transgene (whole brain expression of the transgene could not be detected), but this must not be disregarded as a possibility with respect to some metabolic effects. The failure of the transgenic mice to increase in weight on exposure to a high-fat diet is one such instance.
Does targeted beta cell expression of c-Kit present any potential adverse effects? A rapid drop in blood glucose was observed in response to food deprivation, and this could present a problem in conditions of scant food supply. The potential danger of an unbalanced beta cell mass was recently discussed [21] in the context of tissue-specific repression of Pdgfra, as well as other growth-stimulating genes [22]. Perhaps restrained c-Kit expression in beta cells is a mechanism of protection against episodes of hypoglycaemia between meals owing to excess insulin.

Ideally, targeted beta cell-specific gene transfer of c-Kit seems an attractive strategy for treating imbalances in glucose homeostasis. However the technology as it stands at present does not allow such manipulation and therefore alternative strategies must be considered. The presently available option is using a molecular inhibitor of GSK3 $\beta$ such as 1-azakenpaullone to achieve ameliorating effects but this is an unsatisfactory strategy, since GSK3 $\beta$ has multiple roles in diverse organs. Further delineation of the precise signalling cascade converging at GSK $3 \beta$ may help to identify a key component(s) more specific for the beta cell that could serve as a druggable target for the treatment of type 2 diabetes. However, species differences between humans and rodents should also be taken into account, since human beta cells show a much lower capacity for replication in adulthood [23] than rodent cells; thus, the anticipated effect of cKit may, in such a case, become insignificant. On the other hand, if the insulinotropic effect prevails despite modest or undetectable beta cell replication in human beta cells, c-Kit activation may still be considered a relevant strategy for developing novel treatment regimens.

Acknowledgements The author is grateful to A. Tengholm and N. Welsh (Department of Medical Cell Biology, Uppsala University, Uppsala, Sweden) for comments and suggestions.

Funding The commentary was supported by grants from the Swedish Research Council (no. 54X-10822), the Swedish Cancer Foundation (no. 100494), the Swedish Diabetes Fund (no. DIA 2010-003) and the Family Ernfors Fund.

Duality of interest The author declares no competing interest.

Contribution statement The author conceived, drafted and approved this commentary.

\section{References}

1. Ronnstrand L (2004) Signal transduction via the stem cell factor receptor/c-Kit. Cell Mol Life Sci 61:2535-2548

2. Betsholtz C (2004) Insight into the physiological functions of PDGF through genetic studies in mice. Cytokine Growth Factor Rev 15:215-228 
3. Feng ZC, Li J, Turco BA, Riopel M, Yee SP, Wang R (2012) Critical role of c-Kit in beta cell function: increased insulin secretion and protection against diabetes in a mouse model. Diabetologia. doi:10.1007/s00125-012-2566-5

4. Swenne I, Heldin CH, Hill DJ, Hellerstrom C (1988) Effects of platelet-derived growth factor and somatomedin-C/insulin-like growth factor I on the deoxyribonucleic acid replication of fetal rat islets of Langerhans in tissue culture. Endocrinology 122:214 218

5. Welsh M, Claesson-Welsh L, Hallberg A et al (1990) Coexpression of the platelet-derived growth factor (PDGF) B chain and the PDGF $\beta$ receptor in isolated pancreatic islet cells stimulates DNA synthesis. Proc Natl Acad Sci U S A 87:5807-5811

6. LeBras S, Czernichow P, Scharfmann R (1998) A search for tyrosine kinase receptors expressed in the rat embryonic pancreas. Diabetologia 41:1474-1481

7. Chen H, Gu X, Liu Y et al (2011) PDGF signalling controls agedependent proliferation in pancreatic beta-cells. Nature 478:349355

8. Oberg C, Waltenberger J, Claesson-Welsh L, Welsh M (1994) Expression of protein tyrosine kinases in islet cells: possible role of the Flk-1 receptor for beta-cell maturation from duct cells. Growth Factors 10:115-126

9. Oberg-Welsh C, Welsh M (1996) Effects of certain growth factors on in vitro maturation of rat fetal islet-like structures. Pancreas $12: 334-339$

10. Rachdi L, El Ghazi L, Bernex F, Panthier JJ, Czernichow P, Scharfmann R (2001) Expression of the receptor tyrosine kinase KIT in mature beta-cells and in the pancreas in development. Diabetes 50:2021-2028

11. Yashpal NK, Li J, Wang R (2004) Characterization of c-Kit and nestin expression during islet cell development in the prenatal and postnatal rat pancreas. Dev Dyn 229:813-825

12. Li J, Goodyer CG, Fellows F, Wang R (2006) Stem cell factor/cKit interactions regulate human islet-epithelial cluster proliferation and differentiation. Int J Biochem Cell Biol 38:961-972
13. Li J, Quirt J, Do HQ et al (2007) Expression of c-Kit receptor tyrosine kinase and effect on beta-cell development in the human fetal pancreas. Am J Physiol Endocrinol Metab 293:E475-E483

14. Krishnamurthy M, Ayazi F, Li J et al (2007) c-Kit in early onset of diabetes: a morphological and functional analysis of pancreatic betacells in c-KitW-v mutant mice. Endocrinology 148:5520-5530

15. Feng ZC, Donnelly L, Li J, Krishnamurthy M, Riopel M, Wang R (2012) Inhibition of Gsk $3 \beta$ activity improves $\beta$-cell function in $c-\mathrm{Kit}^{\mathrm{Wv} /+}$ male mice. Lab Invest 92:543-555

16. Garcia-Ocana A, Vasavada RC, Cebrian A et al (2001) Transgenic overexpression of hepatocyte growth factor in the beta-cell markedly improves islet function and islet transplant outcomes in mice. Diabetes 50:2752-2762

17. Welsh M, Christmansson L, Karlsson T, Sandler S, Welsh N (1999) Transgenic mice expressing Shb adaptor protein under the control of rat insulin promoter exhibit altered viability of pancreatic islet cells. Mol Med 5:169-180

18. Bernal-Mizrachi E, Wen W, Stahlhut S, Welling CM, Permutt MA (2001) Islet $\beta$ cell expression of constitutively active Akt1/PKB $\alpha$ induces striking hypertrophy, hyperplasia, and hyperinsulinemia. $\mathrm{J}$ Clin Invest 108:1631-1638

19. Liu Y, Tanabe K, Baronnier D et al (2010) Conditional ablation of Gsk-3 $\beta$ in islet beta cells results in expanded mass and resistance to fat feeding-induced diabetes in mice. Diabetologia 53:2600-2610

20. Grant SF, Thorleifsson G, Reynisdottir I et al (2006) Variant of transcription factor 7-like 2 (TCF7L2) gene confers risk of type 2 diabetes. Nat Genet 38:320-323

21. Schuit F, van Lommel L, Granvik M et al (2012) $\beta$-cell-specific gene repression: a mechanism to protect against inappropriate or maladjusted insulin secretion? Diabetes 61:969-975

22. Thorrez L, Laudadio I, van Deun K et al (2011) Tissue-specific disallowance of housekeeping genes: the other face of cell differentiation. Genome Res 21:95-105

23. Perl S, Kushner JA, Buchholz BA et al (2010) Significant human beta-cell turnover is limited to the first three decades of life as determined by in vivo thymidine analog incorporation and radiocarbon dating. J Clin Endocrinol Metab 95:E234-E239 